\title{
Periodontite materna e baixo peso ao nascer: revisão sistemática e metanálise
}

\author{
Maternal periodontitis and low birth weight: \\ systematic review and meta-analysis
}

Edla Carvalho Lima Porto (https://orcid.org/0000-0003-1664-6511) ${ }^{1}$ Isaac Suzart Gomes Filho (https://orcid.org/0000-0002-4270-8491) ${ }^{1}$ Josicélia Estrela Tuy Batista (https://orcid.org/0000-0003-3826-3570) ${ }^{1}$ Amanda Oliveira Lyrio (https://orcid.org/0000-0001-7740-2524) ${ }^{2}$ Elivan Silva Souza (https://orcid.org/0000-0001-5143-2362) ${ }^{2}$ Ana Claudia Morais Godoy Figueiredo (https://orcid.org/0000-0002-9892-1531) ${ }^{2}$ Mauricio Gomes Pereira (https://orcid.org/0000-0001-9512-3502) ${ }^{2}$

Simone Seixas da Cruz (https://orcid.org/0000-0003-2828-0887) ${ }^{3}$

${ }^{1}$ Universidade Estadual de Feira de Santana. Av. Transnordestina s/n, Feira de Santana. 44036-900 Novo Horizonte BA Brasil. edlaclporto@gmail.com ${ }^{2}$ Universidade de Brasília. Brasília DF Brasil.

${ }^{3}$ Universidade Federal do

Recôncavo da Bahia. Santo

Antônio de Jesus BA Brasil.
Abstract Recent evidence points to the influence of periodontal inflammatory processes on the occurrence of low birth weight. Although many studies employed robust investigation methods, there is still no general agreement on the relationship between maternal periodontitis and low birth weight. The search for studies was conducted until April 2019. The studies included cohort and case-control studies that estimated the association between periodontitis and low birth weight, with no restriction on language or date of publication. Analysis of the heterogeneity of studies, subgroup analyses and meta-analyses with a random effects model were performed. Summary association measurements were estimated using the crude and adjusted Odds Ratio, with respective 95\% confidence intervals. Visual inspection of graphs was used to assess publication bias. A total of 21 articles were identified, all of which were selected for the meta-analysis. The final model indicates that periodontitis in pregnant women was associated with low birth weight $\left(O R_{\text {gross }}=2.13\right.$; $C I 95 \%=1.60-2.83 ; I^{2}=80.0 \%$ and $O R_{\text {adjusted }}=2.64$; $\left.C I 95 \%=2.04-3.42 ; I^{2}=17.4 \%\right)$. Pregnant women with periodontitis may be more than twice as $l i-$ kely to have low birth weight babies.

Key words Periodontitis, Low birth weight, Systematic Review, Meta-analysis
Resumo Evidências recentes apontam para a influência de processos inflamatórios periodontais na ocorrência de baixo peso ao nascer. Embora muitos estudos empregaram métodos robustos de investigação, ainda não existe consenso sobre o tópico. Analisar sistematicamente a relação entre a periodontite materna e o baixo peso ao nascer. A busca por estudos foi realizada até abril de 2019. Os delineamentos de estudos incluídos foram coorte e caso-controle que estimaram a associação entre a periodontite e o baixo peso ao nascer, sem limite quanto ao idioma ou data da publicação. Análise de heterogeneidade dos estudos, análises de subgrupo e metanálises com modelo de efeitos randômicos foram realizadas. Foram estimadas as medidas de associação sumária por meio da Odds Ratio bruta e ajustada, com respectivos intervalos de confiança a 95\%. A inspeção visual de gráficos foi empregada para avaliar viés de publicação. Um total de 21 artigos foram identificados e todos foram selecionados para a metanálise. O modelo final aponta que a periodontite em gestantes se associou ao baixo peso ao nascer $\left(O R_{\text {bruta }}=2,13\right.$; $I C 95 \%=1,60-2,83 ; I^{2}=80,0 \%$ e OR $R_{\text {ajustada }}=2,64$; $\left.I C 95 \%=2,04-3,42 ; I^{2}=17,4 \%\right)$. Gestantes com periodontite podem ter mais que o dobro de probabilidade de terem filhos com baixo peso ao nascer. Palavras-chave Periodontite, Baixo peso ao nascer, Revisão Sistemática, Metanálise 


\section{Introdução}

O baixo peso ao nascer representa um dos principais determinantes da mortalidade infantil, recém-nascidos com essa condição tem um risco de morrer 40 vezes maior, quando comparados àqueles que nascem com peso normal ${ }^{1,2}$. Esse desfecho gestacional indesejável atua como um fator deletério ao desenvolvimento mental e físico da criança, com potenciais danos à vida adulta e elevados custos para o serviço de saúde ${ }^{3-6}$.

Entre os fatores de risco mais conhecidos para o baixo peso ao nascer, apontam-se aqueles ligados diretamente à menor duração da gestação, ao retardo de crescimento intrauterino, ou a combinação de ambos. Evidências recentes, ainda inconclusivas, mostraram a influência de processos inflamatórios na ocorrência de baixo peso ao nascer. Por essa razão fatores que potencialmente estimulam a carga inflamatória sistêmica materna têm sido investigados ao longo de duas décadas, a exemplo da periodontite ${ }^{7-11}$.

A presença dos microrganismos no biofilme dental pode ativar o sistema imune elevando a produção de macrófagos, linfócitos B e T, desencadeando uma resposta imunológica exacerbada. Estudos descreveram que esse quadro inflamatório tem relação com efeitos sistêmicos e particularmente para as gestantes, pode haver redução do peso ao nascer ${ }^{12-15}$.

Estima-se que, mundialmente, 3,9 milhões de pessoas são afetadas por doenças orais ${ }^{16}$. Dados mundiais da frequência de periodontite em gestantes são escassos, porém, no Brasil a prevalência deste agravo varia de $11 \%{ }^{17}$ a $47 \%{ }^{18}$. Aliado a isso, cerca de $15 \%$ a $20 \%$ de todos os nascimentos, no mundo, são categorizados como de baixo peso $^{19}$, sendo que destes, $95,6 \%$ ocorrem nos países em desenvolvimento ${ }^{20}$, tornando evidente a importância de políticas públicas que combatam esses problemas, evitando assim eventos adversos ao binômio mãe-filho ${ }^{21}$.

Todavia, a hipótese de associação entre periodontite e baixo peso ao nascer ainda representa um forte dissenso entre estudiosos ${ }^{22-25}$. Justificando esforços da comunidade científica para elucidar as questões que permanecem obscuras em torno da relação de causalidade sob análise. Recentemente, muitas investigações têm sido realizadas sobre o tema ${ }^{26-32}$, ao mesmo tempo em que o método epidemiológico tem se aperfeiçoado, influenciando na qualidade do método desses estudos.

Neste sentido, este estudo teve o objetivo de avaliar as evidências científicas existentes em tor- no da associação entre periodontite materna e baixo peso ao nascer, por meio de uma revisão sistemática com metanálise de estudos observacionais.

\section{Método}

\section{Registro e protocolo}

Uma busca de revisões sistemáticas com metanálise foi realizada sobre o tema "Associação entre a periodontite em gestantes e baixo peso ao nascer" na base International Prospective Register of Systematic Reviews (PROSPERO) e não foram encontrados registros de metanálise com essa temática específica.

Para o relato desta pesquisa, foram empregados Preferred Reporting Items for Systematic Reviews and Meta-Analyses - The PRISMA Statement $^{33} \mathrm{e}$ o Meta-analysis of Observational Studies in Epidemology (MOOSE) ${ }^{34}$.

\section{Critérios de elegibilidade}

Os critérios de elegibilidade consistiram em estudos do tipo coorte e caso-controle que avaliaram a relação da periodontite em gestantes e o baixo peso ao nascer e forneceram ou permitiram o cálculo das medidas de odds ratio (OR).

Não houve restrição em relação à data de publicação ou ao idioma utilizado. $\mathrm{O}$ baixo peso ao nascer foi definido quando o recém-nascido apresentava peso inferior a $2.500 \mathrm{~g}^{19}$. Os artigos que informaram exclusivamente medidas agregadas de prematuridade e baixo peso ao nascer, bem como estudos que não apresentaram ou não permitiram o cálculo da medida de associação foram excluídos. Foram também excluídas investigações cujo poder foi inferior a $80 \%$. Além disso, as investigações que apresentaram como diagnóstico de periodontite apenas os descritores clínicos periodontais ou diagnóstico autorreferido também foram excluídas.

\section{Fontes de informação}

A busca por informações foi realizada até o dia 06 de abril de 2019. As bases eletrônicas utilizadas foram: Medline (via PubMed), Web of Science, Embase, Scopus, Lilacs e SciELO. As listas de referências dos artigos selecionados para a revisão sistemática foram examinadas, além de bases de dados específicas que contém textos da literatura cinzenta como dissertações ou teses 
(ProQuest e Portal de Periódicos CAPES/MEC Coordenação de Aperfeiçoamento de Pessoal de Nível Superior/Ministério da Educação).

\section{Estratégias de busca}

Os descritores utilizados e seus sinônimos foram identificados no Medical Subject Headings $(\mathrm{MeSH})$ e no Descritores em Ciência da Saúde (DeCS). Foram empregados unitermos e operadores booleanos, além de outros similares, na tentativa de se obter uma estratégia de busca sensível. A avaliação da qualidade da frase de busca foi realizada por meio do instrumento Peer Review of Electronic Search Strategies (PRESS) ${ }^{35}$.

\section{Seleção dos estudos}

Após a exclusão de duplicatas por meio do programa State of the Art through Systematic Review (START), versão 3.3 beta, duas revisoras de forma independente fizeram a seleção dos títulos e resumos (ECLP e JETB). A leitura completa das publicações foi realizada por duas avaliadoras independentes e aquelas que obedeceram aos critérios de elegibilidade foram definitivamente incluídas. Para avaliação da concordância entre as revisoras foi aplicado o índice Kappa ${ }^{36}$. Nos casos em que houve divergência entre as pesquisadoras, a mediação para inclusão das publicações foi realizada por uma outra revisora (SSC).

\section{Extração dos dados}

A extração dos dados das publicações incluídas foi realizada por três pesquisadoras independentes (AOL, ECLP e JETB) e as informações foram confrontadas. Os dados foram sumarizados em um quadro confeccionado no programa Word (Microsoft Office 2016) com os seguintes campos: nome dos autores, ano de publicação, periódico publicado, país, objetivo, delineamento de estudo, local e período da coleta, tamanho amostral, critérios para o diagnóstico da periodontite, principais resultados e presença de variáveis de confundimento/modificadores de efeito.

\section{Avaliação da qualidade dos estudos}

A avaliação da qualidade dos estudos foi realizada de forma independente pelas revisores (AOL, ECLP e JETB) com o emprego do instrumento Newcastle-Ottawa ${ }^{37}$. Os artigos que obtiveram pontuação acima de 5 foram considerados de qualidade moderada e àqueles que apresenta- ram mais que 7 pontos foram classificados com alta qualidade.

\section{Análise dos dados}

Foi elaborada uma síntese dos dados qualitativos dos estudos incluídos na metanálise acerca da periodontite e baixo peso ao nascer.

A heterogeneidade estatística foi mensurada por meio do i-quadrado $\left(\mathrm{I}^{2}\right)$, sendo adotada a classificação de Higgins e Green ${ }^{38}$. De acordo com essa classificação os valores do $\mathrm{I}^{2}$ até $60 \%$ foram considerados como moderados.

As medidas de associação estimadas foram odds ratio e seus respectivos intervalos de confiança de 95\%, obtidas com a metanálise empregando modelos de efeitos randômicos, por meio do método de Der Simonian-Laird. Os achados dos estudos de coorte apresentados em risco relativo foram convertidos em odds ratio.

$\mathrm{O}$ viés de publicação foi analisado por meio da inspeção visual do gráfico em funil (Begg's funnel plot). O gráfico de Galbraith foi empregado para avaliação da fonte de heterogeneidade entre os estudos ${ }^{39}$.

De acordo com a magnitude da heterogeneidade, foi realizada análise de subgrupo segundo o tamanho da amostra, tomando como ponto de corte a mediana. Para identificação da fonte de heterogeneidade, o gráfico de Galbraith foi elaborado e os estudos que se apresentaram como outliers foram excluídos. Finalmente, foi estimada a medida de associação global entre periodontite e baixo peso ao nascer, seu respectivo intervalo de confiança e a heterogeneidade residual.

\section{Resultados}

A busca nos bancos de dados eletrônicos identificou 1.453 registros. Apenas $21^{8,10,11,23,40-57}$ artigos atenderam aos critérios de elegibilidade dessa metanálise (Figura 1). A concordância entre as revisoras na fase de seleção dos estudos foi considerada excelente de acordo com o índice Kappa obtido $(0,79)^{36}$.

A amostra foi constituída por 8.561 gestantes, com mediana de, aproximadamente, 340 mulheres. As características dos artigos selecionados estão descritas na Tabela 1. Do total de 21 artigos incluídos, 16 (76,2\%) tinham delineamento de estudo caso-controle e $5(23,8 \%)$ eram coorte. A maioria dos estudos foi desenvolvido na América do Sul (47,6\%). Em relação aos critérios utilizados para o diagnóstico de periodontite, houve a 


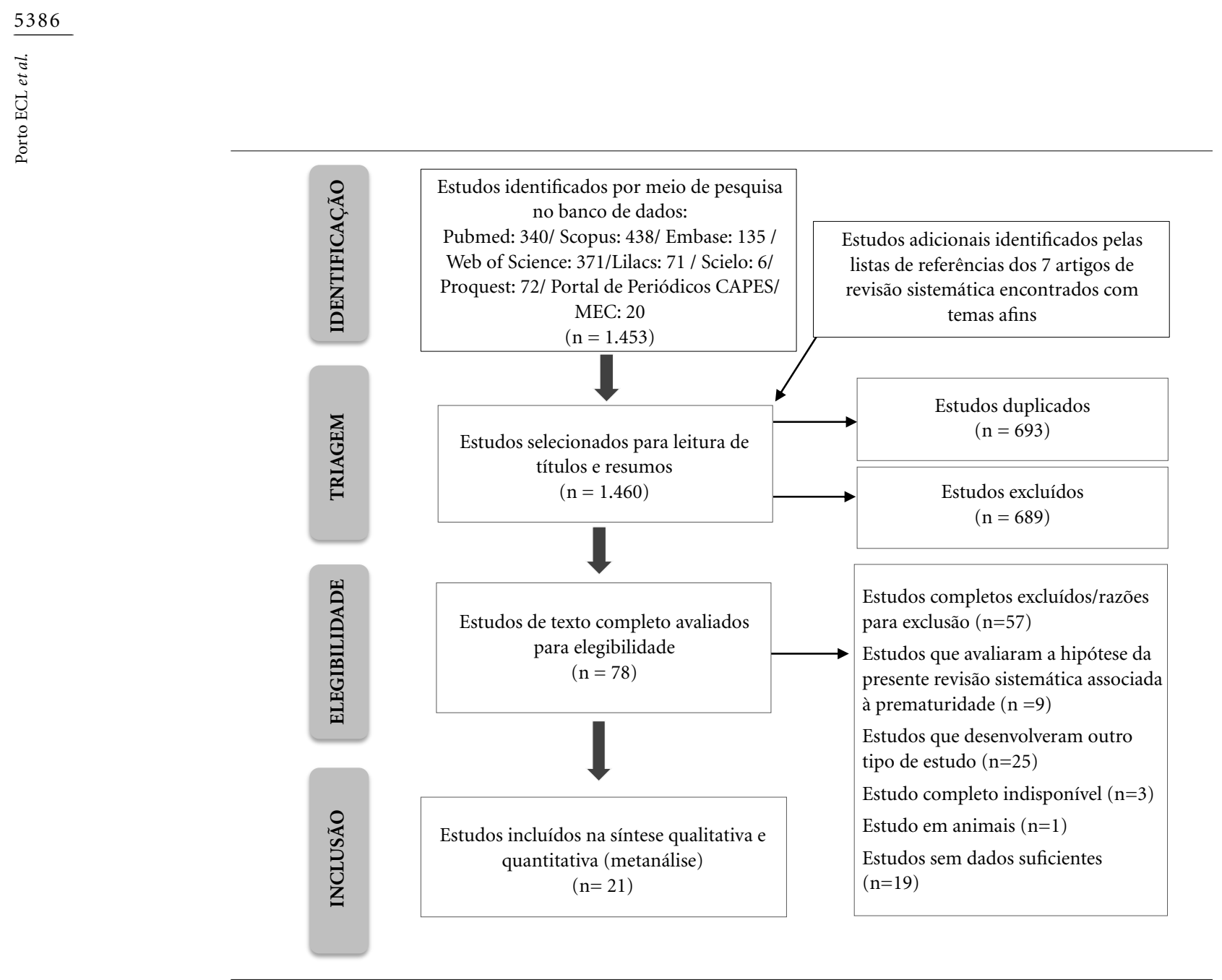

Figura 1. Fluxograma da revisão sistemática da literatura.

Fonte: Elaborado pelos autores.

maior frequência de $23,8 \%$ do critério de Gomes-Filho et al..$^{58}$, seguido do critério da Organização Mundial da Saúde ${ }^{59}$ com 14,3\%. O período de publicação da maioria dos artigos foi entre 2011 e $2018(66,7 \%)$ e, mais da metade recebia algum tipo de financiamento $(57,1 \%)$.

Os resultados da metanálise (Figura 2) sumarizaram $\mathrm{OR}_{\text {bruta }}=2,13$ (IC95\%: 1,60-2,83), porém com elevada heterogeneidade $\left(\mathrm{I}^{2}=80 \%\right)$.

Dentre os 21 artigos incluídos inicialmente na metanálise, apenas 16 deles apresentaram medidas de associação ajustadas ${ }^{8,10,11,40,42-51,55,60}$. A medida global ajustada foi de 2,33 (IC95\% $=1,66$ $3,27)$, com heterogeneidade elevada $\left(\mathrm{I}^{2}=74,6 \%\right)$, conforme apresentado na Figura 3. Após a exclusão de $5 \operatorname{artigos}^{10,42,45,51,60}$, por serem considerados a fonte de heterogeneidade desta metanálise (Figura 4), a associação positiva foi mantida e a heterogeneidade foi classificada como baixa $\left(\mathrm{OR}=2,64 ; \mathrm{IC} 95 \%=2,04-3,42 ; \mathrm{I}^{2}=17,4 \%\right)$. Após a inspeção do gráfico em funil, foi considerada baixa a possibilidade de viés de publicação.
A avaliação da qualidade metodológica mostrou que a pontuação dos 21 artigos teve mediana de 9 e média de 8,24 ( $\pm 0,94$ desvio padrão - DP) pontos, apresentando limite inferior de 7 e superior de 9 pontos. Empregando apenas as medidas ajustadas, a qualidade metodológica apresentou mediana de 9 e média de $8,50( \pm 0,73 \mathrm{DP})$ pontos. Ao se analisar apenas os artigos que compuseram a Figura 4 (medidas ajustadas e sem os outliers), as medidas de tendência central da pontuação dos estudos foram: mediana de 9 e média de 8,55 $( \pm 0,69 \mathrm{DP})$.

\section{Discussão}

Os principais resultados desta investigação evidenciaram que gestantes com periodontite tem mais que o dobro da chance de gerarem recémnascido de baixo peso ao nascer. Os achados da presente pesquisa corroboraram revisões sistemáticas com metanálise sobre o tema que inclu- 
Tabela 1. Características gerais dos estudos empregados na metanálise.

\begin{tabular}{|c|c|c|}
\hline Característica & $\mathbf{N}$ & $\%$ \\
\hline \multicolumn{3}{|l|}{ Tipo de desenho de estudo } \\
\hline Caso-controle & 16 & 76,19 \\
\hline Coorte & 5 & 23,81 \\
\hline \multicolumn{3}{|c|}{ Região geográfica de realização do estudo } \\
\hline América & 10 & 47,62 \\
\hline Ásia & 6 & 28,57 \\
\hline Europa & 3 & 14,29 \\
\hline Oceania & 1 & 4,76 \\
\hline África & 1 & 4,76 \\
\hline \multicolumn{3}{|c|}{$\begin{array}{l}\text { Critério utilizado para o diagnóstico da } \\
\text { Periodontite }\end{array}$} \\
\hline Gomes-Filho et al. $(2007)^{58}$ & 5 & 23,82 \\
\hline $\begin{array}{l}\text { Organização Mundial da Saúde } \\
(1997)^{59}\end{array}$ & 3 & 14,29 \\
\hline Lopez et al. (2002) - adaptado ${ }^{68}$ & 2 & 9,53 \\
\hline Bassani et al. $(2007)^{45}$ & 1 & 4,76 \\
\hline Ainamo e Bay $(1975)^{69}$ & 1 & 4,76 \\
\hline Sanchez et al. $(2007)^{70}$ & 2 & 9,52 \\
\hline Offenbacher et al. $(1998)^{71}$ & 1 & 4,76 \\
\hline Carlos et al. $(1986)^{72}$ & 1 & 4,76 \\
\hline Kumar et al. $(2013)^{50}$ & 1 & 4,76 \\
\hline Jacob $(2014)^{44}$ & 1 & 4,76 \\
\hline Madianos et al. $(2001)^{73}$ & 1 & 4,76 \\
\hline Komara $(2016)^{57}$ & 1 & 4,76 \\
\hline Canhota $(2008)^{74}$ & 1 & 4,76 \\
\hline \multicolumn{3}{|l|}{ Tamanho da amostra } \\
\hline$\leq 340$ & 11 & 52,38 \\
\hline$>340$ & 10 & 47,62 \\
\hline \multicolumn{3}{|l|}{ Qualidade metodológica dos estudos } \\
\hline Moderada: 5-6 pontos & 1 & 4,76 \\
\hline Alta: 7-9 pontos & 20 & 95,24 \\
\hline \multicolumn{3}{|l|}{ Ano de publicação do estudo } \\
\hline $2001-2010$ & 7 & 33,33 \\
\hline $2011-2018$ & 14 & 66,67 \\
\hline \multicolumn{3}{|c|}{$\begin{array}{l}\text { Emprego de fatores confundidores na } \\
\text { análise }\end{array}$} \\
\hline Sim & 16 & 76,19 \\
\hline Não & 5 & 23,81 \\
\hline \multicolumn{3}{|l|}{ Financiamento do estudo } \\
\hline Sim & 9 & 42,86 \\
\hline Não relatado & 12 & 57,14 \\
\hline \multicolumn{3}{|c|}{$\begin{array}{l}\text { Emprego de fatores modificadores de } \\
\text { efeito na análise }\end{array}$} \\
\hline Sim & 2 & 9,52 \\
\hline Não & 19 & 90,48 \\
\hline
\end{tabular}

Fonte: Elaborado pelos autores.

íram outros desfechos gestacionais indesejáveis, além do baixo peso ${ }^{26-32}$. No entanto, a necessida- de dessa atualização sobre o tópico ocorreu, uma vez que a última revisão sistemática com metanálise encontrada foi publicada em 2016, com o período de busca dos estudos até janeiro de $2015^{29}$. Além disso, não foi localizada nenhuma revisão sistemática que abordasse o baixo peso ao nascer, exclusivamente como desfecho.

Os resultados dos 21 estudos individuais, incluídos na presente investigação, podem ser considerados conflitantes, vez que um quarto aponta que não há associação. Tais divergências justificam, em parte, a alta heterogeneidade estatística, a qual tende a reduzir a validade da síntese de evidência. Outra potencial fonte de heterogeneidade se refere ao diagnóstico acerca da presença de periodontite, vez que há dificuldade para padronização do diagnóstico dessa enfermidade em gestantes $^{32,61,62}$.

O supracitado argumento é apoiado por estudo $^{58}$ que empregou quatro critérios diagnósti$\cos$ da referida doença e avaliou a associação de acordo com a definição empregada. A magnitude da associação reduziu em função do maior rigor dos critérios, ou seja, as definições diagnósticas com elevada sensibilidade apresentaram maiores medidas de associação, quando comparadas àquelas com especificidade mais expressiva. Nesse sentindo, é necessário a padronização de critérios para determinação da periodontite destinado às gestantes, que vise maior homogeneidade metodológica entre os estudos e um direcionamento mais consistente para prática clínica dos cirurgiões-dentistas.

A diversidade de modelos conceituais adotados para explicar a associação entre periodontite e baixo peso ao nascer também foi uma constatação nessa metanálise. Dentre os 16 estudos que tiveram suas medidas de associação ajustadas para confundimento, apenas dois ${ }^{48,60}$ utilizaram as mesmas covariáveis. Desse modo, avaliar de forma mais consistente os possíveis confundidores é mandatório para neutralizar o efeito espúrio da hipótese investigada e aumentar a qualidade da evidência sobre o tema.

Além disso, a multicausalidade envolvida na ocorrência tanto da exposição, periodontite, quanto do desfecho, baixo peso ao nascer, tem origem em inúmeras características, tais como biológica, comportamental, social e econômica ${ }^{63}$. Por exemplo, sabe-se que a periodontite começa com uma inflamaçãoo da gengiva, oriunda da disbiose do biofilme bacteriano com o hospedeiro, podendo se estender aos tecidos ao redor do dente e, consequentemente, ocasionar a perda dentária. Esse processo envolve a produção de 


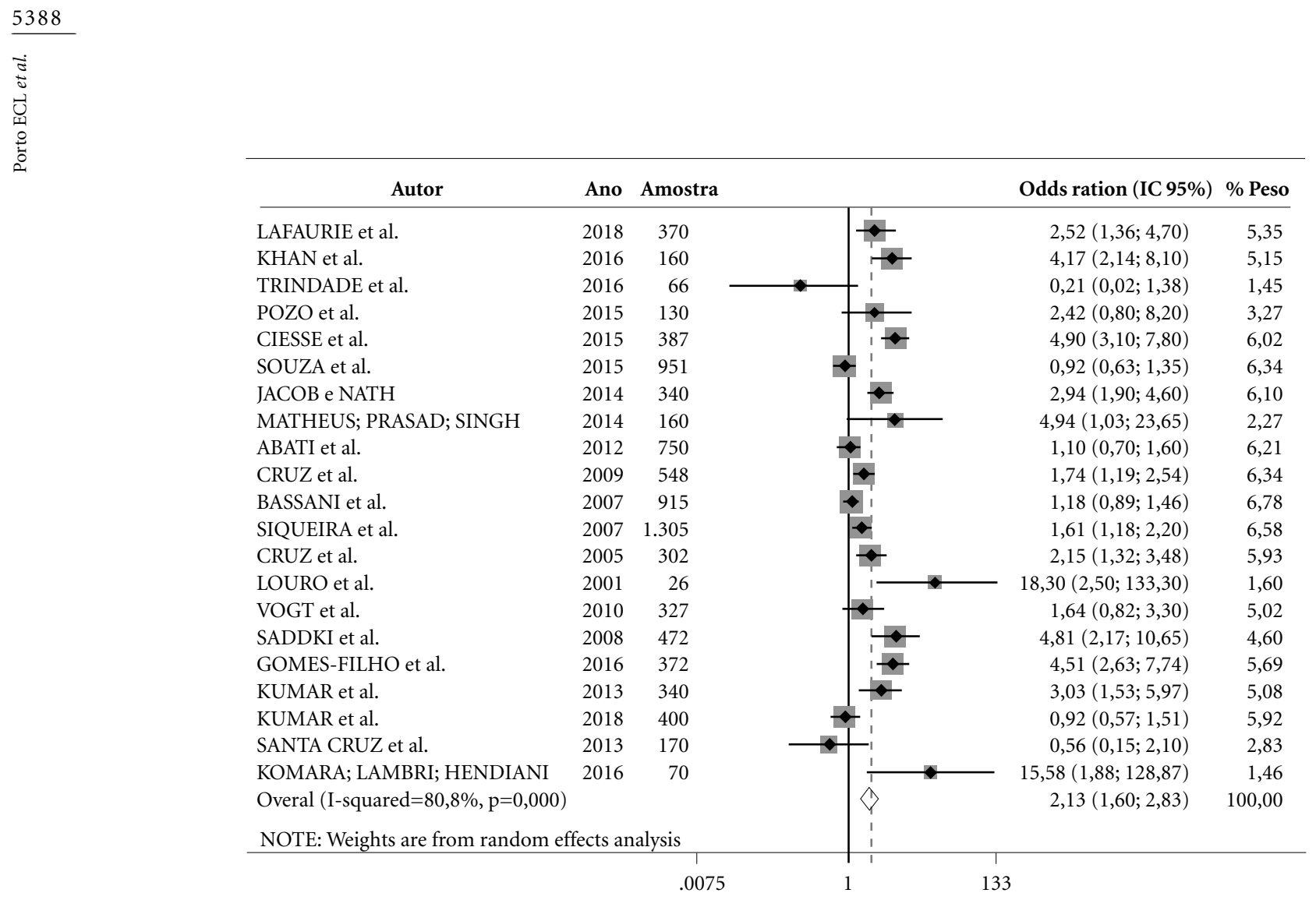

Figura 2. Gráfico forest plot da metanálise com medidas de associação bruta e intervalos de confiança a 95\% dos estudos avaliados.

Fonte: Elaborado pelos autores.

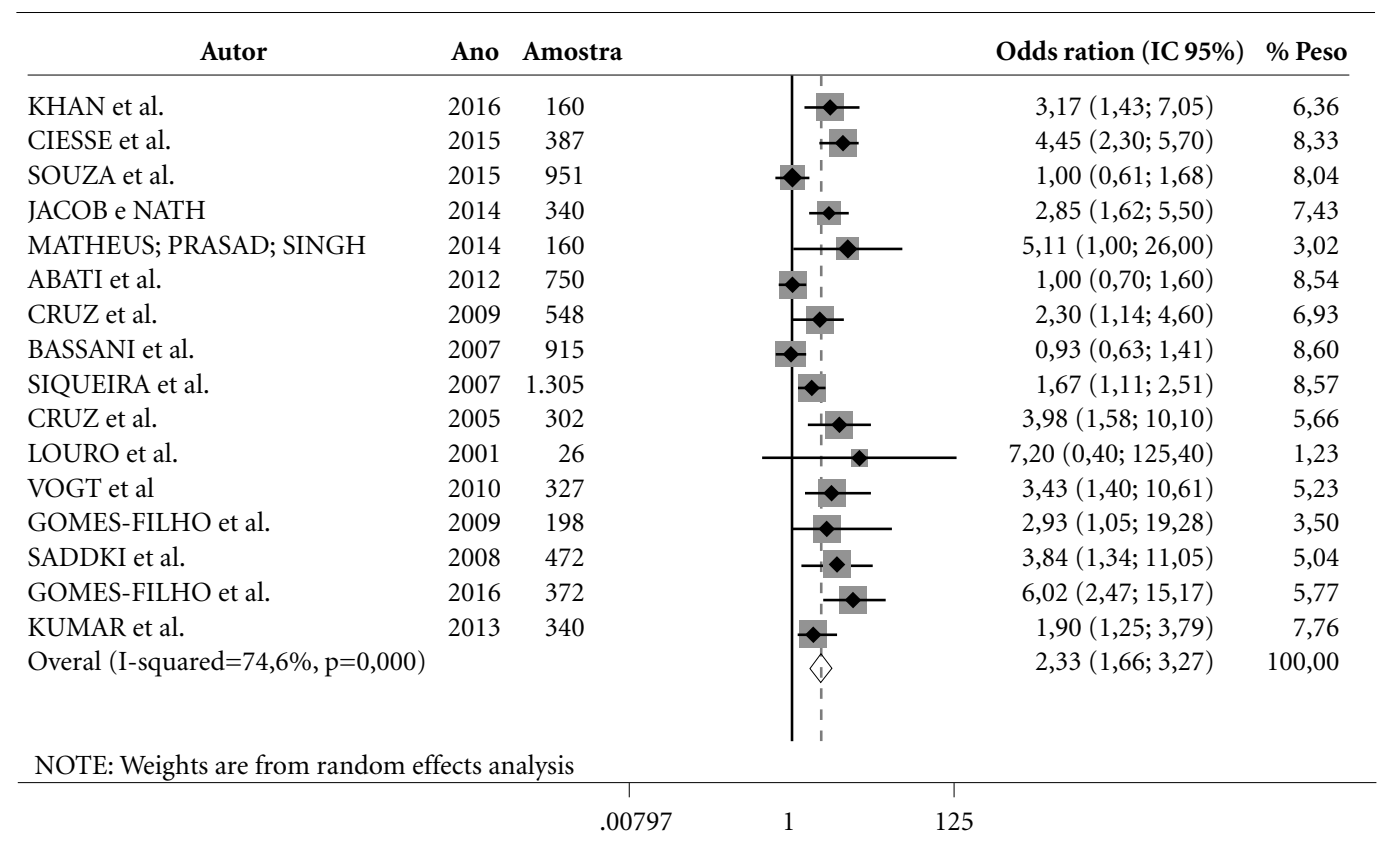

Figura 3. Gráfico forest plot da metanálise com medidas de associação ajustadas e intervalos de confiança a 95\% dos estudos avaliados.

Fonte: Elaborado pelos autores. 
mediadores inflamatórios como as citocinas, interleucina 1 (IL-1), IL-6 e fator de necrose tumoral - TNF, que influenciam à distância no desfecho. Para isso, outros fatores de risco contribuem para essa referida associação $0^{64,65}$.

Ademais, a distribuição geográfica também pode ter influenciado na heterogeneidade dos estudos incluídos na presente revisão sistemática, uma vez que quase metade das pesquisas foram realizadas no continente americano. Sabe-se que os países da América apresentam uma grande disparidade socioeconômica, especialmente àqueles localizados na América do Sul ${ }^{66}$. Essa questão pode produzir impacto negativo ao acesso das mulheres grávidas aos serviços públicos, bem como na prevenção e promoção à saúde bucal materna.

Em relação a qualidade metodológica dos estudos, esta teve seus achados alterados de acordo com a análise de sensibilidade. Ao se comparar a totalidade de artigos incluídos com a análise de sensibilidade, uma melhor qualidade metodológica para os artigos que permaneceram nos modelos finais foi observada (Figura 4). Uma limitação, que merece destaque nessa metanálise, refere-se a inclusão apenas de estudos observacionais, os quais tem maior vulnerabilidade a vieses $^{67}$, embora, em sua grande maioria nesta revisão, os estudos foram classificados como de alta qualidade metodológica.

O emprego de instrumentos reconhecidos internacionalmente para avaliação da qualidade metodológica dos estudos ${ }^{37}$, refinamento das estratégias de busca ${ }^{35}$ e o uso de guias durante o processo de redação ${ }^{33,34}$ representam uma fortaleza da presente revisão sistemática. Ainda, optouse pela utilização de medidas ajustadas para elevar a qualidade da evidência da metanálise. A adoção de técnicas analíticas robustas foi outro aspecto relevante, a exemplo da análise de sensibilidade, padronização de todas as medidas de associação em odds ratio, avaliação crítica das pesquisas consideradas outliers e viés de publicação. Além disso, a definição do desfecho apenas como o baixo peso ao nascer se deveu ao conhecimento da dificuldade de se estabelecer de forma precisa a idade gestacional, normalmente feita por diferentes critérios clínicos não padronizados, que podem interferir nos achados finais, comprometendo a validade interna dos estudos originais, que poderia implicar vieses importantes no estudo de síntese.

Na tentativa de minimizar a possibilidade de viés de publicação foram selecionadas oito bases de dados, sendo duas da literatura cinzenta. Tais bancos contêm pesquisas científicas realizadas em todos os continentes, que mostra a amplitude de captação de textos publicados acerca da periodontite e baixo peso ao nascer.

Por fim, os achados desse estudo indicaram que a periodontite materna é um fator importante para o baixo peso ao nascer, havendo necessidade de maior debate em torno dessa hipótese, especialmente entre os tomadores de decisão. As revisões sistemáticas encontradas sobre o tópico mostraram a necessidade da atualização. No entanto, para elevar a qualidade da evidência, suge-

\begin{tabular}{|c|c|c|c|c|c|c|}
\hline Autor & Ano & Amostra & & & Odds ration (IC 95\%) & $\%$ Peso \\
\hline KHAN et al. & 2016 & 160 & $\rightarrow-$ & & $3,17(1,43 ; 7,05)$ & 8,79 \\
\hline JACOB e NATH & 2014 & 340 & $\rightarrow$ & & $2,85(1,62 ; 5,50)$ & 13,44 \\
\hline MATHEUS; PRASAD; SINGH & 2014 & 160 & + & & $5,11(1,00 ; 26,00)$ & 2,40 \\
\hline CRUZ et al. & 2009 & 548 & $\rightarrow-$ & & $2,30(1,14 ; 4,60)$ & 10,95 \\
\hline SIQUEIRA et al. & 2007 & 1.305 & $\leftarrow$ & & $1,67(1,11 ; 2,51)$ & 23,06 \\
\hline CRUZ et al. & 2005 & 302 & $\rightarrow$ & & $3,98(1,58 ; 10,10)$ & 6,79 \\
\hline LOURO et al. & 2001 & 26 & 1 & & $7,20(0,40 ; 125,40)$ & 0,80 \\
\hline VOGT et al & 2010 & 327 & $\rightarrow$ & & $3,43(1,40 ; 10,61)$ & 5,80 \\
\hline SADDKI et al. & 2008 & 472 & $\stackrel{b}{\longrightarrow}$ & & $3,84(1,34 ; 11,05)$ & 5,40 \\
\hline GOMES-FILHO et al. & 2016 & 372 & 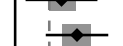 & & $6,02(2,47 ; 15,17)$ & 7,05 \\
\hline KUMAR et al. & 2013 & 340 & $\rightarrow-$ & & $1,90(1,25 ; 3,79)$ & 15,50 \\
\hline \multicolumn{3}{|c|}{ Overal (I-squared $=17.4 \%, \mathrm{p}=0,278)$} & $\Delta$ & & $2,64(2,04 ; 3,42)$ & 100,00 \\
\hline \multicolumn{3}{|c|}{ NOTE: Weights are from random effects analysis } & 1 & & & \\
\hline & 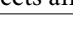 & 1 & & 1 & & \\
\hline & & .00797 & 1 & 125 & & \\
\hline
\end{tabular}

Figura 4. Gráfico forest plot da metanálise com medidas de associação ajustadas e intervalos de confiança a 95\%, sem os estudos considerados outliers. 
re-se a elaboração de uma revisão sistemática de ensaios clínicos randomizados com alta precisão e baixa heterogeneidade, envolvendo exclusivamente o desfecho em questão, para inferências mais robustas.

\section{Referências}

1. Watkins WJ, Kotecha SJ, Kotecha S. All-Cause Mortality of Low Birthweight Infants in Infancy, Childhood, and Adolescence: Population Study of England and Wales. PLOS Med 2016; 13(5):e1002018.

2. Institute of Medicine Committee on Understanding Premature Birth and Assuring Healthy. The National Academies Collection: Reports funded by National Institutes of Health. In: Behrman RE, Butler AS. Preterm Birth: Causes, Consequences, and Prevention. Washington, D.C.: National Academies Press (US) National Academy of Sciences; 2007.

3. Rüdiger M, Heinrich L, Arnold K, Druschke D, Reichert J, Schmitt J. Impact of birthweight on healthcare utilization during early childhood-a birth cohort study. BMC Pediatrics 2019; 19(1):69.

4. Thanh NX, Toye J, Savu A, Kumar M, Kaul P. Health service use and costs associated with low birth weight - a population level analysis. J Pediatr 2015; 167(3):551-556.

5. Govindaraju P, Venugopal S, Shivakumar MA, Sethuraman S, Ramaiah SK, Mukundan S. Maternal periodontal disease and preterm birth: A case-control study. J Indian Soc Periodontol 2015; 19(5):512-515.

6. Gera I. The association between maternal periodontal health and adverse pregnancy outcomes. Orvosi Hetilap 2018; 159(25):999-1007.

7. Kaur M, Geisinger ML, Geurs NC, Griffin R, Vassilopoulos PJ, Vermeulen L, Haigh S, Reddy MS. Effect of intensive oral hygiene regimen during pregnancy on periodontal health, cytokine levels, and pregnancy outcomes: a pilot study. J Periodontol 2014; 85(12):1684-1692.

8. Cruz SS, Costa MCN, Gomes Filho IS, Rezende EJC, Barreto ML, Santos CAST, Vianna MIP, Passos JS, Cerqueira EMM. Contribution of periodontal disease in pregnant women as a risk factor for low birth weight. Community Dent Oral Epidemiol 2009; 37(6):527-533.

\section{Colaboradores}

ECL Porto: planejamento da pesquisa, auxiliou na coleta de dados e redação do artigo. IS Gomes Filho: auxiliou no planejamento da pesquisa e na redação do artigo. JET Batista: auxiliou na coleta de dados e redação do artigo. AO Lyrio: auxiliou na coleta de dados e redação do artigo. ES Souza: auxiliou na redação do artigo. ACMG Figueiredo: auxiliou na análise estatística e redação do artigo. SS Cruz: planejamento da pesquisa, auxiliou na análise estatística e redação do artigo. MG Pereira: auxiliou na redação do artigo.

9. Cruz SS, Costa MC, Gomes-Filho IS, Barreto $\mathrm{ML}$, Santos CAST, Martins AG, Passos JS, Freitas COT, Sampaio FP, Cerqueira EMM. Periodontal therapy for pregnant women and cases of low birthweight: an intervention study. Pediatr Int 2010; 52(1):57-64.

10. Souza LM, Cruz SS, Gomes-Filho IS, Barreto ML, Passos-Soares JS, Trindade SC, Figueiredo ACMG, Alves CMC, Coelho JMF, Vianna MIP. Effect of maternal periodontitis and low birth weight-A case control study. Acta Odontol Scand 2016; 74(1):73-80.

11. Cruz SS, Costa MCN, Gomes Filho IS, Vianna MIP, Santos CT. Doença periodontal materna como fator associado ao baixo peso ao nascer. Rev Saude Publica 2005; 39:782-787.

12. Puertas A, Magan-Fernandez A, Blanc V, Revelles L, O’Valle F, Pozo E, León R, Mesa F. Association of periodontitis with preterm birth and low birth weight: a comprehensive review. J Matern Fetal Neonatal Med 2018; 31(5):597-602.

13. Cardoso EM, Reis C, Manzanares-Céspedes MC. Chronic periodontitis, inflammatory cytokines, and interrelationship with other chronic diseases. Postgrad Med 2018; 130(1):98-104.

14. Hegde R, Awan K. Effects of periodontal disease on systemic health. Dis Month 2019; 65(6):185-192.

15. Cintra LTA, Estrela C, Azuma MM, Queiroz ÍOA, Kawai T, Gomes-Filho JE. Endodontic medicine: interrelationships among apical periodontitis, systemic disorders, and tissue responses of dental materials. Braz Oral Res 2018; 32(Supl. 1):e68.

16. Richards D. Oral diseases affect some 3.9 billion people. Evid Based Dent 2013; 14(2):35.

17. Piscoya MDBV, Ximenes RAA, Silva GM, Jamelli SR, Coutinho SB. Periodontitis-associated risk factors in pregnant women. Clinics 2012; 67(1):27-33. 
18. Vogt M, Sallum AW, Cecatti JG, Morais SS. Factors associated with the prevalence of periodontal disease in low-risk pregnant women. Reproductive Health 2012; 9(1):3.

19. World Health Organisation (WHO). Global Nutrition Targets 2025: Low birth weight policy brief. Genebra: WHO; 2014

20. Mahumud RA, Sultana M, Sarker AR. Distribution and determinants of low birth weight in developing countries. J Prev Med Public Health 2017; 50(1):18-28.

21. Costa RS, Caldevilla DE, Gallo PR, Sena BF, Leone C. Incidência e características dos recém-nascidos de peso insuficiente de uma coorte de neonatos de um hospital público regional de área metropolitana. J Hum Growth Develop 2013; 23(2):238-244.

22. Krüger MSdM, Casarin RP, Pinto GS, Pappen FG, Camargo MBJ, Correa FOB, Romano AR. Maternal periodontal disease and adverse perinatal outcomes: is there an association? A hospital-based case-control study. J Matern Fetal Neonatal Med 2019; 32(20):3401-3407.

23. Lafaurie GI, Gómez LA, Montenegro DA, Avila J, Tamayo MC, Lancheros MC, Quiceno J, Trujillo TG, Noriega LA, Grueso ML, Cepeda K. Periodontal condition is associated with adverse perinatal outcomes and premature rupture of membranes in low-income pregnant women in Bogota, Colombia: a case-control study. J Matern Fetal Neonatal Med 2020; 33(1):16-23.

24. Chanomethaporn A, Chayasadom A, Wara-Aswapati N, Kongwattanakul K, Suwannarong W, Tangwanichgapong K, Sumanonta G, Matangkasombut O, Dasanayake AP, Pitiphat W. Association between periodontitis and spontaneous abortion: A case-control study. J Periodontol 2019; 90(4):381-390.

25. Rangel-Rincón LJ, Vivares-Builes AM, Botero JE, Agudelo-Suárez AA. An Umbrella Review Exploring the Effect of Periodontal Treatment in Pregnant Women on the Frequency of Adverse Obstetric Outcomes. $J$ Evid Based Dent Pract 2018; 18(3):218-239.

26. Corbella S, Taschieri S, Francetti L, De Siena F, Del Fabbro M. Periodontal disease as a risk factor for adverse pregnancy outcomes: a systematic review and meta-analysis of case-control studies. Odontology 2012; 100(2):232-240

27. Ide M, Papapanou PN. Epidemiology of association between maternal periodontal disease and adverse pregnancy outcomes--systematic review. J Periodontol 2013; 84(Supl. 4):S181-S194.

28. Daalderop L, Wieland B, Tomsin K, Reyes L, Kramer BW, Vanterpool SF, Been JV. Periodontal disease and pregnancy outcomes: overview of systematic reviews. JDR Clin Trans Res 2018; 3(1):10-27.

29. Corbella S, Taschieri S, Del Fabbro M, Francetti L, Weinstein R, Ferrazzi E. Adverse pregnancy outcomes and periodontitis: A systematic review and meta-analysis exploring potential association. Quintessence Int 2016; 47(3):193-204.

30. Vergnes JN, Sixou M. Preterm low birth weight and maternal periodontal status: a meta-analysis. Am J Obstet Gynecol 2007; 196(2):135.e131-137.

31. Khader YS, Ta'ani Q. Periodontal diseases and the risk of preterm birth and low birth weight: a meta-analysis. J Periodontol 2005; 76(2):161-165.
32. Chambrone L, Guglielmetti MR, Pannuti CM, Chambrone LA. Evidence grade associating periodontitis to preterm birth and/or low birth weight: I. A systematic review of prospective cohort studies. J Clin Periodontol 2011; 38(9):795-808.

33. Liberati A, Altman DG, Tetzlaff J, Mulrow C, Gøtzsche PC, Ioannidis JPA, Clarke M, Devereaux PJ, Kleijnen J, Moher D. The PRISMA Statement for Reporting Systematic Reviews and Meta-Analyses of Studies That Evaluate Health Care Interventions: Explanation and Elaboration. PLoS Med 2009; 6(7):e1000100.

34. Stroup DF, Berlin JA, Morton SC, Olkin I, Williamson GD, Rennie D, Moher D, Becker BJ, Sipe TA, Thacker SB. Meta-analysis of observational studies in epidemiology: a proposal for reporting. Meta-analysis Of Observational Studies in Epidemiology (MOOSE) group. JAMA 2000; 283(15):2008-2012.

35. McGowan J, Sampson M, Salzwedel DM, Cogo E, Foerster V, Lefebvre C. PRESS Peer Review of Electronic Search Strategies: 2015 Guideline Statement. J Clin Epidemiol 2016; 75:40-46.

36. COCHRANE. Identifying and measuring heterogeneity [Internet]. 2019 [acessado 2018 maio 18]. Disponível em: https://handbook-5-1.cochrane.org/chapter_9/9_5_2_identifying_and_measuring_heterogeneity.htm.

37. Wells G. The Newcastle-Ottawa Scale (NOS) for assessing the quality of nonrandomised studies in meta-analysis [Internet]. [acessado 2018 maio 18]. Disponível em: http://www, ohri. ca/programs/clinical_epidemiology.oxford.htm.2004.

38. Higgins J, Green S. Manual Cochrane de revisiones sistemáticas de intervenciones. Cochrane; 2011.

39. Dinnes J, Deeks J, Kirby J, Roderick P. A methodological review of how heterogeneity has been examined in systematic reviews of diagnostic test accuracy. Health Technol Assess 2005; 9(12):1-113.

40. Khan NS, Ashraf RN, Noor S, ur-Rahman M, Mashhadi SF, Rashid Z, Sajjad F, Nazar AF, Nazar HS, Syed R. Association of maternal periodontitis with low birth weight in newborns in a tertiary care hospital. J Ayub Med Coll Abbottabad 2016; 28(1):120-125.

41. Trindade SC, Santos RR, Gomes Filho IS, Soares JSP, Cruz SS, Rezende EJC, Mameluque S, Dias VO, Souza Júnior EB, Cerqueira EMM, Meyer R, Barreto ML. Periodontite e baixo peso ao nascer-Um estudo piloto no Município de Montes Claros/MG, Brasil. Rev Saude Colet UEFS 2016; 6(2):43-50.

42. Cisse D, Diouf M, Faye A, Diadhiou M, Tal-Dia A. Periodontal disease of pregnant women and low weight Newborn in Senegal: a case-control study. Open J Epidemiol 2015; 5(1):1.

43. Mathew RJ, Bose A, Prasad J, Muliyil J, Singh D. Maternal periodontal disease as a significant risk factor for low birth weight in pregnant women attending a secondary care hospital in South India: a case-control study. Indian J Dental Res 2014; 25(6):742.

44. Jacob PS, Nath S. Periodontitis among poor rural Indian mothers increases the risk of low birth weight babies: a hospital-based case control study. J Periodontal Implant Sci 2014; 44(2):85-93.

45. Bassani D, Olinto M, Kreiger N. Periodontal disease and perinatal outcomes: a case-control study. J Clin Periodontol 2007; 34(1):31-39. 
46. Siqueira FM, Cota LOM, Costa JE, Haddad JPA, Lana ÂMQ, Costa FO. Intrauterine growth restriction, low birth weight, and preterm birth: adverse pregnancy outcomes and their association with maternal periodontitis. J Periodontol 2007; 78(12):2266-2276.

47. Louro PM, Fiori HH, Louro F, Steibel J, Fiori RM. Periodontal disease in pregnancy and low birth weight. $J$ Pediatr 2001; 77(1):23-28.

48. Vogt M, Sallum AW, Cecatti JG, Morais SS. Periodontal disease and some adverse perinatal outcomes in a cohort of low risk pregnant women. Reprod Health 2010; 7:29.

49. Saddki N, Bachok N, Hussain NHN, Zainudin SLA, Sosroseno W. The association between maternal periodontitis and low birth weight infants among Malay women. Community Dent Oral Epidemiol 2008; 36(4):296-304.

50. Kumar A, Basra M, Begum N, Rani V, Prasad S, Lamba AK, Verma M, Agarwal S, Sharma S. Association of maternal periodontal health with adverse pregnancy outcome. J Obstet Gynaecol Res 2013; 39(1):40-45.

51. Abati S, Villa A, Cetin I, Dessole S, Lugliè PF, Strohmenger L, Ottolenghi L, Campus GG. Lack of association between maternal periodontal status and adverse pregnancy outcomes: a multicentric epidemiologic study. J Matern Fetal Neonatal Med 2013; 26(4):369372.

52. Kumar A, Sharma DS, Verma M, Lamba AK, Gupta MM, Sharma S, Perumal V. Association between periodontal disease and gestational diabetes mellitus-A prospective cohort study. J Clin Periodontol 2018; 45(8):920-931.

53. Pérez B MG, Pérez MF, Araque L, Rincón Á. Peridontitis crónica en mujeres embarazadas y el nacimiento de niños de bajo peso y parto pretérmino. Acta Odontol Venez 2014; 52:1.

54. Pozo E, Mesa F, Ikram MH, Puertas A, Torrecillas -Martínez L, Ortega-Oller I, Magán-Fernández A, Rodríguez-Martínez MD, Padial-Molina M, SánchezFernández E, Galindo-Moreno P, O’Valle F. Preterm birth and/or low birth weight are associated with periodontal disease and the increased placental immunohistochemical expression of inflammatory markers. Histol Histopathol 2016; 31(2):231-237.

55. Gomes-Filho IS, Pereira EC, Cruz SS, Adan LFF, Vianna MIP, Passos-Soares JS, Trindade SC, Oliveira EP, Oliveira MT, Cerqueira EMM, Pereira AL, Barreto ML, Seymour GJ. Relationship Among Mothers' Glycemic Level, Periodontitis, and Birth Weight. J Periodontol 2016; 87(3):238-247.

56. Santa Cruz I, Herrera D, Martin C, Herrero A, Sanz M. Association between periodontal status and preterm and/or low-birth weight in Spain: clinical and microbiological parameters. J Periodontal Res 2013; 48(4):443-451.

57. Komara I, Lambri S, Hendiani I. Relationship Between Periodontal Disease Index And Low Birth Weight Babies In Pregnant Women With Periodontitis. IJIHS 2016; 4(1):15-19.

58. Gomes-Filho IS, Cruz SS, Rezende EJ, Santos CAST, Soledade KR, Magalhães MA, Azevedo ACO, Trindade SC, Vianna MIP, Passos JS, Cerqueira EMM. Exposure measurement in the association between periodontal disease and prematurity/low birth weight. J Clin Periodontol 2007; 34(11):957-963.
59. World Health Organization (WHO). Oral Health Surveys: Basic Methods. 4a ed. Geneva: WHO; 1997.

60. Gomes-Filho IS, Cruz SS, Passos JS, Figueiredo ACMG, Souza LM. Avaliação prospectiva da periodontite materna e baixo peso ao nascer. Rev Periodontia 2009; 19(4):121-128.

61. Polyzos NP, Polyzos IP, Mauri D, Tzioras S, Tsappi M, Cortinovis I, Casazza G. Effect of periodontal disease treatment during pregnancy on preterm birth incidence: a metaanalysis of randomized trials. Am J Obstet Gynecol 2009; 200(3):225-232.

62. Uppal A, Uppal S, Pinto A, Dutta M, Shrivatsa S, Dandolu V, Mupparapu M. The effectiveness of periodontal disease treatment during pregnancy in reducing the risk of experiencing preterm birth and low birth weight: a meta-analysis. J Am Dent Assoc 2010; 141(12):1423-1434.

63. Van Dyke TE, Sheilesh D. Risk factors for periodontitis. J Int Acad Periodontol 2005; 7(1):3-7.

64. Slots J. Periodontitis: facts, fallacies and the future. $P e$ riodontol 2000 2017;75(1):7-23.

65. Ameet MM, Avneesh HT, Babita RP, Pramod PM. The relationship between periodontitis and systemic diseases - hype or hope? J Clin Diagn Res 2013; 7(4):758-762.

66. Noronha KV, Andrade MV. Social inequality in health and the utilization of health services among the elderly in Latin America. Rev Panam Salud Publica 2005; 17(5-6):410-418.

67. Rothman K, Greenland S, Lash T. Epidemiologia Moderna. $3^{\mathrm{a}}$ ed. Porto Alegre: Artmed Editora; 2016.

68. Lopez NJ, Smith PC, Gutierrez J. Periodontal Therapy may reduce the risk of preterm low birth weight in women with periodontal disease: a randomized controlled trial. J Periodontol 2002; 73(8):911-924.

69. Ainamo J, Bay I. Problems and proposals for recording gingivitis and plaque. Int Dent $J$ 1975; 25(4):229-235.

70. Sanchez AR, Bagniewski S, Weaver AL, Vallejos N. Correlations between Maternal Periodontal Conditions and Preterm Low Birth Weight Infants. J Int Acad Periodontol 2007; 9(2):34-41.

71. Offenbacher S, Beck JD, Lieff S, Slade G: Role of periodontitis in systemic health: spontaneous preterm birth. J Dent Educ 1998; 62(10):852-858.

72. Carlos JP, Wolf MD, Kingman A. The extend and severity index: as simple method for use in epidemiologic studies of periodontal disease. J Clin Periodontol 1986; 13(5):500-504.

73. Madianos PN, Lieff S, Murtha AP, Boggess KA, Auten Jr RL, Beck JD, Offenbacher S. Maternal periodontitis and prematurity part II: maternal infection and fetal exposure. Ann Periodontol 2001; 6(1):175-182.

74. Canhota C. Qual a importância do estudo piloto? In: Silva EE, organizador. Investigação passo a passo: perguntas e respostas para investigação clínica. Lisboa: APMCG; 2008. p. 69-72.

Artigo apresentado em 29/02/2020

Aprovado em 20/04/2020

Versão final apresentada em 22/04/2020

Editores-chefes: Romeu Gomes, Antônio Augusto Moura da Silva 